\title{
Evaluación de los problemas que limitan el impacto de la extensión pública en el oriente de Guatemala
}

\author{
Assessment of the problems that limit the impact of the public extension \\ service in eastern Guatemala
}

\author{
Fernando Pablo Landini ${ }^{1}$ (D), Gilda Luciana Vargas² (D) \\ ${ }^{1}$ Consejo Nacional de Investigaciones Científicas y Técnicas (CONICET), Universidad de la Cuenca del Plata Posadas, Misiones, \\ Argentina. E-mail: landini_fer@hotmail.com \\ 2Universidad de Buenos Aires, Buenos Aires, Argentina. E-mail: luciana0003@hotmail.com
}

Cómo citar: Landini, F. P., \& Vargas, G. L. (2020). Evaluación de los problemas que limitan el impacto de la extensión pública en el oriente de Guatemala. Revista de Economia e Sociologia Rural, 58(1), e192529.

https://doi.org/10.1590/1806-9479.2020.192529

\begin{abstract}
Resumen: Los sistemas de extensión rural públicos juegan un rol central en la seguridad alimentaria, y más aún en un país como Guatemala, que cuenta con un $46,6 \%$ de niños menores de cinco años con desnutrición crónica. En este trabajo se exponen los resultados de un estudio orientado a comprender los problemas que limitan los resultados y el impacto del servicio de extensión del Ministerio de Agricultura, Ganadería y Alimentación (Maga) en el oriente del país. Se realizaron 12 entrevistas individuales y 4 grupos focales con extensionistas de la institución en los departamentos de Chiquimula y Zacapa, las cuales fueron analizadas a partir de un proceso de codificación realizado con el apoyo del software Atlas Ti. La muestra y los departamentos fueron seleccionados por razones de accesibilidad y conveniencia. Se concluye que el entorno de politización y corrupción política tiene un papel estructurante en la dinámica del servicio de extensión del Maga, que el sistema de extensión del Maga es sumamente débil a nivel institucional, que existen problemas significativos relacionados con los recursos humanos, y que las prácticas de extensión tienen impacto limitado. Se recomienda generar acciones para fortalecer el sistema de extensión del Maga y diseñar estrategias creativas para formar extensionistas.
\end{abstract}

Palabras clave: extensión rural, instituciones públicas, evaluación, Guatemala.

Abstract: Public rural extension systems play a key role in food security, and even more in a country such as Guatemala, wherein $46.6 \%$ of children under five years old suffer malnutrition. In this paper, the results of study aimed at understanding in a systemic way the problems that limit the results and impact of the extension service of the Ministry of Agriculture, Husbandry and Alimentation (Maga) in eastern Guatemala are presented. In this vein, 12 individual interviews and 4 focus groups with Maga's rural extensionists were conducted in the departments of Chiquimula and Zacapa, which were analyzed based on a codification process carried out with the support of Atlas Ti software. The departments and the research participants for reasons of accessibility and convenience. Results show that the context of politicization and political corruption structure the dynamic of the Maga's extension system, that the Maga's extension service is institutionally weak, that there are relevant problems in the area of human resources, and that extension practices have low impact. We recommend generating actions to strengthen the Maga's extension system, and designing creative strategies to train rural extensionists.

Keywords: rural extension, public institutions, assessment, Guatemala.

\section{Introducción}

En la actualidad existe un fuerte consenso respecto del importante rol que juegan los sistemas de extensión rural (ER) en los procesos de desarrollo e innovación rural (Leeuwis, 2004; Sulaiman \& Davis, 2012; Zwane, 2012), y en reducción de desigualdades (Belik, 2015). En esta línea, se observa que los paradigmas para pensar los sistemas de ER han evolucionado 
desde enfoques tradicionales centrados en la difusión de tecnologías, hacia abordajes territoriales y sistémicos en los cuales se reconoce la existencia de una multiplicidad de actores rurales con los que debe trabajarse de manera horizontal y articulada, para impulsar procesos de desarrollo (Sæther, 2010).

En el contexto de este cambio paradigmático, diversos autores han señalado la necesidad de impulsar sistemas de ER plurales, caracterizados por la existencia de una diversidad de proveedores que tengan la capacidad de responder a las necesidades de diferentes perfiles de productores (Knierim et al., 2017). Esta perspectiva, si bien reconoce la necesidad de servicios de ER privados, también destaca el rol del Estado en la provisión de bienes públicos, como la sustentabilidad ambiental, y como apoyo a los sectores rurales más empobrecidos (Anderson \& Feder, 2004; Klerkx et al., 2006). Así, se reconoce la potencialidad de la ER pública como herramienta para la seguridad alimentaria (Baiardi \& Alencar, 2014; Forrest, 2017), lo que resulta central en Guatemala, país que cuenta con un $46,6 \%$ de niños menores de cinco años con desnutrición crónica (Food and Agriculture Organization, 2017).

El sistema público de ER guatemalteco fue creado durante la década del '1950 con el apoyo de los Estados Unidos (Ortiz et al., 2011). En el marco de las políticas de ajuste que caracterizaron la región en los años ‘1980 y ‘1990, la Dirección General de Servicios Agrícolas (Digesa), dependiente del Ministerio de Agricultura, Ganadería y Alimentación (Maga), expresión de la extensión pública en Guatemala, pierde presupuesto hasta que es definitivamente cerrada a fines de los '1990. Luego, en el marco del resurgimiento de la ER en América Latina (Aguirre, 2012), en el año 2010 el Maga comienza un proceso de apertura de Agencias Municipales de ER (Amer) en todo el país, a partir de un consenso alcanzado entre el gobierno de Guatemala y el sector privado, la academia, y diferentes organismos internacionales de cooperación técnica y financiera (Cárdenas Hernández, 2014)

Pese a la importancia de la recuperación del sistema de ER público en Guatemala, diferentes actores reconocen que este no ha podido consolidarse a lo largo de los años, presentando múltiples debilidades. Atendiendo a la importancia que ha adquirido en los últimos años la evaluación de los servicios de ER, tanto como herramienta de control como de aprendizaje (Christoplos et al., 2012), en este trabajo se presentan los resultados de un estudio orientado a comprender de manera sistémica los problemas que limitan los resultados y el impacto del servicio de ER del Maga en el oriente del país.

\section{Metodología}

Para alcanzar el objetivo propuesto se realizó un estudio cualitativo de carácter exploratorio-descriptivo. Para esto, primero se realizó una revisión documental relativa al Maga y a las políticas de desarrollo rural guatemaltecas. Esto incluyó los siguientes documentos:

- Política Nacional de Desarrollo Rural Integral (PNDRI). Gobierno de Guatemala. 2009.

- Programa de Agricultura Familiar para el Fortalecimiento de la Economía Campesina (PAFFEC) 2016-2020. Gobierno de Guatemala y FAO (sin fecha)

- $\quad$ Cuaderno de extensionistas 3: Marco político-estratégico. Maga. 2014.

- $\quad$ Cuaderno de extensionistas 4: Marco metodológico. Maga. 2014.

- Tesis de maestría titulada: Caracterización del Sistema Nacional de Extensión Rural en Guatemala, departamentos de Chimaltenango, Baja Verapaz y Alta Verapaz. Autora: Jenny Cárdenas Hernández. Centro Agronómico Tropical de Investigación y Enseñanza. 2014.

- Página web del Maga (Guatemala, 2018).

Luego, en julio de 2017 se realizaron 12 entrevistas individuales con extensionistas y 4 grupos focales, incluyendo entrevistas a la Coordinadora de ER y al Coordinador de Capacitación del Maga del departamento de Chiquimula. En total fueron entrevistados 15 hombres y 9 mujeres, 18 del departamento de Chiquimula y 6 del de Zacapa, ambos localizados al oriente del país. La muestra fue intencional. La Fundación Acción contra el Hambre (ACH), que trabaja en la región con el Maga y con otras instituciones en la región, realizó los contactos y coordinó la realización de las entrevistas con los participantes. Como 
requisito para la inclusión en la muestra se estableció que las personas entrevistadas debían desempeñarse como extensionistas en alguna de las AMER del Maga en los departamentos seleccionados, o como sus autoridades de nivel departamental. Se optó por trabajar en estos departamentos y no en otros por razones de conveniencia, en particular la utilidad del conocimiento a desarrollarse para la implementación de acciones de apoyo a la extensión rural por parte de $\mathrm{ACH}$ en la región. Las entrevistas fueron grabadas, previo consentimiento informado de los participantes. Para el análisis, los fragmentos relevantes fueron transcriptos utilizando el aplicativo oTranscribe (2018).

Seguidamente, se analizaron los textos transcriptos a partir de un proceso de codificación y construcción de categorías temáticas (Taylor \& Bodgan, 1987) con el apoyo del software Atlas Ti. Este proceso incluyó tres momentos. En el primero, a partir de los objetivos de este trabajo, se identificaron a nivel teórico dos categorías centrales: 'descripción de la institucionalidad y del trabajo de extensión rural del Maga', y 'problemas para la práctica de extensión', las cuales fueron utilizadas para codificar la totalidad del material. Para la primera categoría se identificaron 101 fragmentos, mientras que para la segunda 146. El segundo momento correspondió a un proceso de subcategorización, que permitió identificar inductivamente subcategorías a partir de las categorías teóricas iniciales, lo que hizo posible describir con detalle las prácticas de los extensionistas y la institucionalidad vinculada, e identificar y describir un listado de problemas específicos. Por último, el tercer paso correspondió a una labor analítica de síntesis o agregación, a partir de la construcción de áreas problemáticas en base a los problemas individuales, y de la identificación de relaciones causales entre ellos. A continuación se presentar los resultados de la investigación.

\section{Resultados}

A fin de presentar los resultados se los divide en dos grandes áreas. Así, primero se presenta una descripción destallada del sistema de ER del Maga y de su funcionamiento, a fin de contextualizar los problemas identificados, para luego sí pasar a un análisis detallado de los problemas y de sus relaciones de retroalimentación.

\subsection{El sistema de ER del Maga}

\subsubsection{Organización del sistema de extensión del Maga}

El Maga cuenta con sedes en los 22 departamentos del país. En cada una hay un Director Departamental, y una serie de unidades o coordinaciones, incluyendo la de Extensión Rural. Las Amer, que dependen de las direcciones departamentales, se ubican una en cada municipio, y tienen al menos tres extensionistas. Los extensionistas pueden ser: Extensionistas de Desarrollo Agropecuario Rural (DAR), Extensionistas para la Agricultura Familiar (AF) y Extensionistas para el Hogar Rural (EHR). Los DAR coordinan las Amer, deben poseer título universitario y les corresponde articular con otras instituciones del territorio. También deben llevar adelante las tareas propias de los AF. Los AF no poseen título universitario, sino que suelen tener formación técnica, muchos de ellos son peritos agrónomos. Ellos deben conformar y brindar capacitación y asesoramiento a grupos de productores denominados Cader (Centros de Aprendizaje para el Desarrollo Rural). Las EHR son mujeres que usualmente poseen título de Maestra de Educación para el Hogar o de licenciadas en Trabajo Social. Su trabajo consiste en dar capacitaciones a mujeres sobre preparación de alimentos, normas de higiene y planificación familiar, entre otros.

Las Amer trabajan con agricultores familiares, particularmente aquellos en situación de pobreza y pobreza extrema. A la vez, organizan su trabajo siguiendo los ejes estratégicos del Programa de Agricultura Familiar para el Fortalecimiento de la Economía Campesina (Paffec): 'Incremento sostenible de producción familiar campesina para la seguridad alimentaria y nutricional', 'Acceso a mercados y cadenas de valor', y 'Desarrollo institucional para la agricultura familiar'. Por último, cabe destacar que el Maga no sólo funciona como actor central de la ER a nivel nacional, sino que también opera como líder e instancia de coordinación del Sistema Nacional de Extensión Rural (SNER). 


\subsubsection{Contratación de extensionistas}

En julio de 2017 el Maga contaba aproximadamente con casi 1500 extensionistas en todo el país. Sin embargo, aproximadamente sólo 100 de ellos tenían puestos permanentes como funcionarios, mientras que el $90 \%$ (o incluso más) corresponderían a puestos de 'consultor' (contratos temporarios). La diferencia entre estos encuadres administrativos es abismal, ya que mientras que los primeros poseen estabilidad laboral, seguro médico, aguinaldo, jubilación, derecho a licencias y vacaciones pagas, los 'consultores' no cuentan con ninguno de estos beneficios. Más aun, los períodos de contratación de los consultores son irregulares y pueden modificarse de año en año. Un extensionista comenta: "en el caso de las ONGs [...] solo una vez firmé [el contrato] nada más, y en el Maga 3 meses, y ahí que descansamos un mes, y que viene el otro mes, de ahí que... 4 meses". En este marco, si bien la mayoría de los extensionistas se mantienen de un período de contratación a otro, hay un porcentaje que se modifica, por razones no explicitadas.

\subsubsection{Formación y capacitación de los extensionistas}

Dependiendo del momento en que ingresaron a la institución, algunos entrevistados comentaron que recibieron una inducción inicial. Otros destacan haber recibido materiales de capacitación publicados por el Maga. Sin embargo, todos los entrevistados indicaron que no existió un periodo inicial propiamente de capacitación o formación. A la vez, los entrevistados también indicaron que, pese a existir una Unidad Departamental de Capacitación, no existe un plan o una estrategia sistemática de capacitación para los extensionistas, fundamentalmente a causa de falta de recursos. Comenta una entrevistada: "falta capacitación, primero capacitar al recurso humano, nosotros como recurso humano estamos abandonados al 100\%". En este contexto, los extensionistas suelen recibir capacitaciones con cierta periodicidad, aunque de manera no permanente, con temas de capacitación variados, que muchas veces dependen de lo que otros actores ofrecen a los extensionistas del Maga, principalmente la cooperación internacional.

\subsubsection{Trabajo de extensión: los Cader y la metodología Campesino a Campesino (CaC)}

EI MAGA propone utilizar la metodología de extensión CaC. Si bien no se trata de una metodología única, existe acuerdo en que se basa en diferentes pilares, entre los que se encuentran el fortalecimiento del intercambio de conocimientos y experiencias entre productores, el aprendizaje práctico/experiencial, y el efecto multiplicador de los nuevos conocimientos generados a partir de la figura del promotor campesino, quien reemplaza al extensionista tradicional (Holt Giménez, 2008).

El Maga operativiza la metodología CaC a partir de la figura del Cader. Los Cader son grupos de productores que comparten problemáticas y se organizan para mejorar su producción en torno a un predio que sirve como espacio de experimentación y aprendizaje. Los Cader cuentan con uno o dos promotores que funcionan como coordinadores, además de recibir el apoyo de un extensionista. Usualmente los Cader tienen entre 15 y 25 integrantes. La cantidad de Cader por municipio es variable, siendo muy importante diferenciar entre aquellos que teóricamente existen y aquellos que se encuentran activos, ya que estos últimos suelen ser muchos menos. En términos generales, los promotores son elegidos por los integrantes de los Cader en base a su capacidad de liderazgo.

Más allá de esto, el modo de trabajo de cada Amer e incluso de cada extensionista con los Cader es muy variable. Teóricamente, los extensionistas construyen anualmente un plan operativo anual (POA) con cada Cader. No obstante, los cambios de extensionistas, la cantidad de Cader, así como la falta de insumos para la producción y la de medios para ir a terreno hacen que muchas veces los POA sean una simple formalidad. Dice un entrevistado: "nosotros como extensionistas cada gobierno hacemos un plan de grupo [...] para cumplir las necesidades que ellos están demandando [...] entonces como extensionista no lo puedo lograr hacer porque no tengo los insumos".

Las EHR suelen hacer capacitaciones con las mujeres o con las esposas de los integrantes de los Cader en su área de trabajo. Los extensionistas que trabajan a nivel productivo (DAR y 
AF) realizan capacitaciones de productores, fundamentalmente de promotores. Aquí, el rol de los promotores será el de aplicar lo aprendido en su predio y replicar las capacitaciones con los integrantes de su propio Cader: "se les capacita a ellos [los promotores], ya ellos llegan con su grupo y lo replican". En este marco, el énfasis en la experimentación activa de la metodología $\mathrm{CaC}$ queda reemplazado por procesos de capacitación a promotores y la expectativa de que estos generen parcelas demostrativas en sus predios.

\subsection{Problemas del Sistema de ER del Maga}

A continuación se presentan los problemas que enfrenta el sistema de ER del Maga. Para esto, se los clasifica tomando como referencia la diferenciación que hace la FAO (Food and Agriculture Organization, 2010) para abordar el desarrollo de capacidades: entorno o contexto, nivel organizacional, y nivel individual. Aun cuando se podría haber usado otro criterio clasificatorio, se opta por este esquema dada su utilidad analítica. A la vez, se agrega un cuarto nivel, diferenciando a nivel individual entre extensionistas y productores.

\subsubsection{Problemas contextuales o del entorno}

Problema 1: Elevado grado de corrupción política en el escenario institucional guatemalteco, lo que lleva a la politización de las acciones de extensión. Desde la perspectiva de los entrevistados, esto refiere a un conjunto de problemas relacionados, entre los cuales se destaca la fuerte incidencia de alcaldes y diputados nacionales en la selección y permanencia de los extensionistas, lo que implica que las personas seleccionadas no necesariamente son aquellas con los mejores antecedentes. De hecho, los extensionistas mencionan que la mejor estrategia para permanecer en el puesto no es tener formación o experiencia sino simplemente tener un 'padrino político'. De esta manera, los extensionistas incorporados terminan estando en deuda con aquellos que los apoyaron. Un entrevistado lo explica: "[entonces nos dicen] 'mirá, se lo vas a dar [la ayuda] a la gente que nosotros queremos', y si no se hace, pues lo sacan a uno [del puesto de extensionista]".

Este elevado grado de incidencia política en el nombramiento de extensionistas también implica que las autoridades políticas cuentan con poder punitivo sobre los extensionistas, particularmente pedir que no les renueven los contratos o que los cambien de municipio en el que trabajan, generando así lugar para nombrar a técnicos de su preferencia. En este contexto, existe una práctica que se llama 'mesa limpia', que implica pedir a las autoridades de extensión que saquen a todos los extensionistas que están trabajando en un municipio para poder participar de la selección de los nuevos.

\subsubsection{Problemas relacionados con el marco institucional}

Problema 2: Debilidad institucional del sistema de extensión rural del Maga. Este problema hace referencia a un conjunto heterogéneo de factores que limitan la capacidad del Maga para actuar de manera sólida y coordinada para alcanzar los objetivos que se propone. Entre ellos cabe mencionar:

+ 2.1. Contratos por períodos cortos y alta rotación de extensionistas. La amplia mayoría de los extensionistas del Maga posee puestos temporarios que se renuevan cada pocos meses. Esto, sumado a los pedidos de los alcaldes de cambiar a extensionistas de sus municipios, lleva a que haya una altísima rotación de personal. Lamentablemente, esto termina reduciendo la eficacia y eficiencia de la labor de los extensionistas, quienes tienen que adaptarse al trabajo cuando ingresan y volver a generar relaciones de confianza con los Cader y con las instituciones del territorio cuando son trasladados, algo que toma meses, e incluso años (Landini, 2016a). Un entrevistado evidencia este problema: "uno ya ganó la confianza [del productor], el cariño de ellos, y que por una cuestión política [...] venga un técnico nuevo y ¿qué pasa?, [los productores dicen] 'ah no, con ese [nuevo] no queremos trabajar', entonces eso es lo que pasa".

+ 2.2. Falta de recursos operativos, vehículos e insumos para trabajo de ER. En general, los recursos para llevar adelante las acciones de extensión son sumamente escasos, incluso inexistentes. Esto limita fuertemente las acciones de extensión y su impacto ya que, si bien 
puede haber disponibilidad de recursos humanos, la falta de recursos materiales hace que su potencialidad no se pueda concretar. Por ejemplo, muchas Amer no cuentan con edificio propio, existiendo casos en los cuales los mismos extensionistas han optado por alquilar locales con su propio salario. Al mismo tiempo, salvo excepciones puntuales derivadas de aportes de la cooperación internacional, las AMER no cuentan ni con vehículos ni con combustible para quien decida usar su propio medio de transporte: "nosotros aquí [...] yo al menos no he tenido combustible [para trabajar] desde julio del año pasado".

+ 2.3. Falta de lineamientos institucionales estables para el trabajo de extensión. Si bien los lineamientos propuestos por el Paffec y la estructura de trabajo a partir de los Cader organizan y ordenan el trabajo de extensión, los extensionistas destacan que las autoridades de distintos niveles cambian constantemente los lineamientos de trabajo, por lo que se termina teniendo escasa continuidad: "viene un gobierno se le ocurre una... [nueva idea] y hay que empezar de cero, hay que borrar todo lo que hicimos anteriormente y comenzar con lo que el gobierno dice".

+ 2.4. Problemas de cobertura y falta de recursos humanos. Pese a que el Maga tiene Amer en todos los municipios, los entrevistados encuentran insuficiente la cantidad de extensionistas para llegar a todos los productores. Esta situación, sumada a la falta de recursos operativos, redunda en que no se dé seguimiento adecuado a todos los Cader.

+ 2.5. Excesiva centralización. Este problema hace referencia a la concentración de la toma de decisiones a nivel central en el Maga, lo que da insuficiente flexibilidad para adaptarlas a los diferentes territorios. Dice un entrevistado "los proyectos vienen de [la capital, ciudad de] Guate[mala] y entonces dice 'tantos sistemas de riego', y el problema [de los productores] es que no hay agua, y entonces, ¿dónde ponerlos si no hay agua?". Al mismo tiempo, la centralización también hace referencia a la escasa descentralización administrativa para la ejecución de fondos a nivel departamental, lo que reduce la flexibilidad para la toma de decisiones.

Problema 3: Generación de una cultura de la simulación. Al encontrarse en una situación de gran precariedad laboral, los extensionistas se sienten en la necesidad de mostrar resultados de su trabajo, aun cuando la institución no ofrezca los medios necesarios para hacerlos posibles. Así, se termina generando una cultura organizacional en la que el Maga hace 'como si' diera los recursos necesarios para hacer el trabajo, y los extensionistas, en algunas oportunidades, terminan encontrando las estrategias para mostrar que hacen su trabajo 'como si' lo hubieran hecho. Los extensionistas deben presentar un informe de trabajo mensual que contenga fotos y firmas de quienes asistieron a las capacitaciones, como prueba de lo realizado. No obstante, hay entrevistados que comentan que las fotos y los listados no siempre son fidedignos, pudiendo mostrar más de lo que realmente se hizo. Comenta una entrevistada: "¿quién evalúa? yo evalúo mi trabajo, yo misma, y si yo quiero reporto lo que realmente hice, y si no... le pongo... agregados".

Problema 4: Pérdida de memoria la institucional y de vínculos establecidos por la rotación de extensionistas. El cambio frecuente de extensionistas tiene consecuencias graves en el trabajo de extensión. El caso extremo es el cambio de todos los extensionistas de un municipio a causa de presiones de los alcaldes, ya que esto hace que se corten procesos y se pierdan los vínculos establecidos tanto con productores como con instituciones. Así, es probable que las acciones deban iniciarse de cero (cuando no hay suficiente registro de las acciones), o al menos que sean difíciles de retomar. Comenta un entrevistado: "eso es lo que provoca que no haya un avance en las comunidades, [...] cuando se contrata nuevo personal, agarra un nuevo grupo, viene alguien más, [se crea otra vez un] nuevo grupo, entonces ¿dónde está el seguimiento del primer grupo que se formó?" A la vez, toda esta situación fomentará que los productores sean cada vez más desconfiados y reticentes con los extensionistas, procurando obtener beneficios cortoplacistas (pidiendo insumos, por ejemplo), al perder confianza en la continuidad del trabajo.

Problema 5. Dificultades para la articulación interinstitucional. Este problema tiene dos facetas, una de nivel territorial y otra en el marco del SNER. A nivel territorial, si bien los extensionistas destacan la importancia de trabajar con otras instituciones, también señalan que esto muchas veces es difícil, porque las prioridades $u$ objetivos pueden ser diferentes, o pueden existir celos relacionados con la visibilidad que tiene cada institución en las acciones 
que se emprenden: "yo tuve la oportunidad de trabajar con [una ONG internacional], pero ellos, a nivel de arriba, no les gusta coordinar con nosotros [como Maga]". A la vez, también se observa una dificultad de articulación interinstitucional en el marco del SNER, particularmente con instituciones de nivel nacional que realizan acciones de extensión, como la Asociación Nacional del Café, o con organismos de investigación, como el Instituto de Ciencia y Tecnología Agrícola.

\subsubsection{Problemas relacionados con los extensionistas}

Problema 6: Pérdida de buenos extensionistas por influencia política. Como resultado de la incidencia política en la contratación de extensionistas, el criterio de capacidad para cumplir con el rol termina pasando a segundo plano, quedando fuera de la institución personas con elevado nivel de experiencia y formación. Un entrevistado comenta la situación: "ha pasado eso, de que la persona que está preparada o que está formada [un político dice] 'esa no me sirve', 'te vas porque tengo que meter [a otra persona]"'

Problema 7: Inestabilidad y fragilidad laboral de los extensionistas. Como se explicó, gran parte de los extensionistas poseen contratos temporarios que en general dependen de padrinos políticos, lo que los pone en una situación de inestabilidad y fragilidad constante: "hacemos un POA a inicios de año, [...] pero al final en mayo ni contratados estábamos".

Problema 8: Pérdida de compromiso e iniciativa de los extensionistas. Son diversas tanto las razones que llevan a los extensionistas a perder la iniciativa y el compromiso, como sus formas de expresión. En general, se observa que hay un conjunto de extensionistas comprometidos con su trabajo, que incluso usan parte de su salario para comprar combustible o insumos, para poder así cumplir con sus obligaciones. Sin embargo, los nombramientos por razones políticas, la fragilidad de sus puestos y la falta de recursos para operativos terminan minando el compromiso y la iniciativa de los extensionistas. Por esto, y aun cuando le gusta el trabajo que hace, un entrevistado comentó: "si tengo una oportunidad igual a la del Maga, me voy". Es que los extensionistas terminan convenciéndose de que nada pueden hacer para mejorar la situación, cayendo en la desesperanza.

Problema 9: Falta de formación de los extensionistas y de un plan de capacitación. La mayor parte de los extensionistas, cuando ingresaron al Maga, no recibieron capacitación para ser extensionistas. A la vez, a nivel departamental o a nivel nacional, tampoco existe un plan organizado o sistemático de capacitación para extensionistas, por lo que las capacitaciones se organizan usualmente según la disponibilidad concreta de capacitadores o las prioridades de los cooperantes. Dice un entrevistado con experiencia en el tema "todo lo aprovechamos [aunque sabemos que] no responde a un plan institucional".

\subsubsection{Problemas vinculados con los agricultores y con los impactos de las políticas de apoyo}

Problema 10: Desconfianza y escasa credibilidad de los extensionistas. Los extensionistas comentan dificultades para conseguir la confianza de los productores, así como pérdida de credibilidad en sus personas y en el Maga, lo que parece vincularse con hechos como la ruptura de procesos por el cambio de extensionistas, la entrega de ayudas con criterio político, o la realización de relevamientos de datos sin que esto redunde en un beneficio para los productores, entre otros.

Problema 11: Actitud asistencialista y cortoplacista de los productores. Los extensionistas comentan que uno de sus problemas más grandes es lo que llaman 'actitud asistencialista' de los productores, lo que implica que éstos no parecen estar interesados en procesos de capacitación y acompañamiento técnico, sino que lo que buscan casi exclusivamente es el acceso a insumos u otro tipo de ayudas materiales. Un extensionista lo explica: "uno le va a dar la capacitación, pero tiene que regalar algo, la semilla, el fertilizante, si no, la gente no llega. '¿Y qué me va a dar, y qué me va a traer, y cuánto me van a dar?' [dicen los productores]". En este contexto, llama la atención que los extensionistas entiendan estas actitudes de los productores como desinterés, sin poder contextualizar que se trata de respuestas que se dan en un marco donde son los propios servicios de extensión los que no pueden ofrecerles un trabajo serio y sistemático de largo plazo. 
Problema 12. Caderes débiles, baja participación e implicación. Otro problema refiere a la debilidad de muchos Cader, aun cuando los extensionistas no siempre identifiquen esto como problema en sí mismo. En este sentido, se observa que hay Cader que dejan de trabajar en conjunto, por lo que se disuelven: "[en algunas comunidades] nos está tocando reiniciar los CADER, porque se fueron quedando fríos". No obstante, y como se indicó, aún los Cader que se consideran activos no suelen funcionar como se desearía, con un promotor que aporte a la capacitación del grupo, e integrantes realmente implicados y comprometidos con la dinámica de trabajo del grupo.

Problema 14. Escaso impacto de la metodología CaC implementada. El trabajo del Maga se apoya en el supuesto de que la metodología CaC utilizada genera los resultados deseados. Sin embargo, como comenta un extensionista "decirle que hemos tenido buenos resultados con eso sería mentirnos a nosotros mismos". En este contexto, lo más problemático se refiere al vínculo de capacitación que debería establecerse entre promotores y el resto de los integrantes del Cader, ya que los entrevistados indican que las capacitaciones de los promotores a los grupos son casi inexistentes y su impacto limitado: "el sistema en cascada llega hasta el promotor, del promotor hacia abajo ya no hay". Así, parecería que los extensionistas sí alcanzan resultados con los promotores, pero no con los integrantes de los grupos. En este sentido, se observa que tienden a persistir en las comunidades problemas como bajo rendimiento de los cultivos y pérdida de fertilidad del suelo, entre otros. Explica un entrevistado: "el promotor [...] hace el esfuerzo de mantener su huerto [...] obviamente son pocos los que han visto, [...] [el cambio] realmente, si vamos al campo, es muy poco"

\subsection{Análisis sistémico de los problemas identificados}

Como se sugirió al momento de describir los problemas, resulta claro que estos no son independientes, sino que se encuentran articulados por medio de relaciones de causalidad. En la Figura 1 se presenta una propuesta gráfica generada por los autores, en la cual se expresan los problemas con rectángulos y las relaciones causales con flechas, como propone la técnica del Modelo Problemático Integrado (Robirosa et al., 1990). Se destaca que muchas de estas relaciones causales fueron sugeridas al momento de presentación de los datos, mientras que otras se hicieron evidentes al momento de analizar los problemas en términos de un sistema.

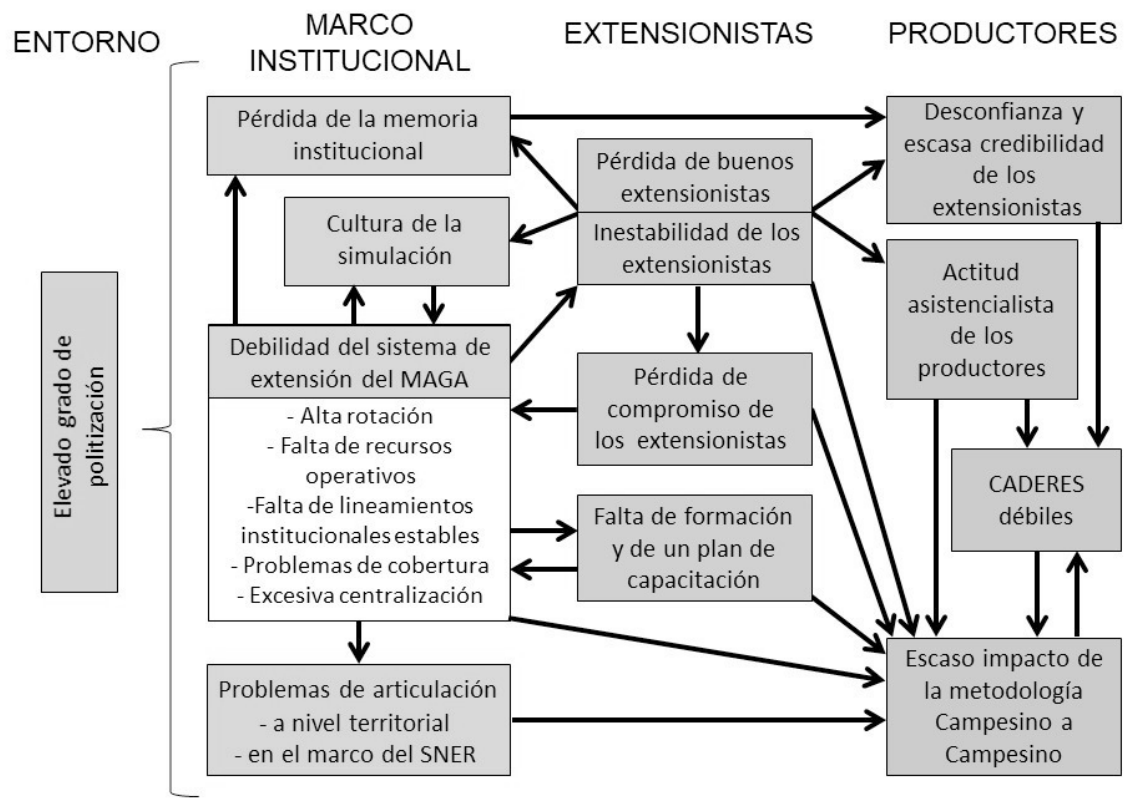

Figura 1. Análisis sistémico de los problemas de la ER del Maga Fuente: Elaboración propia. 
Analizando la Figura 1, se toma conciencia de la fuerza estructurante del entorno político, caracterizado por el clientelismo y la corrupción, en la dinámica del sistema de extensión del Maga, particularmente en lo que hace a su debilidad institucional. A la vez, se observa que existe un conjunto importante de problemas a nivel de los recursos humanos del Maga (extensionistas), que se articulan fuertemente con la debilidad de la institución, como ser inestabilidad y pérdida de buenos extensionistas, establecimiento de una cultura de la simulación, pérdida de compromiso con la tarea y falta de formación. Finalmente, los problemas relacionados con los recursos humanos para llevar adelante el trabajo de extensión, terminan incidiendo negativamente en el trabajo con los productores, generándose actitudes asistencialistas, desconfianza, Caderes débiles y, en definitiva, escaso impacto a nivel de los productores. Así, sin negar la existencia de factores causales con incidencia propia en los diferentes niveles, se observa una relación causal estructurante que parte de la existencia de un elevado grado de corrupción política, la cual da forma a una institución de extensión poco consolidada, con numerosas debilidades, que tiene importantes problemas a nivel de recursos humanos, todo lo que termina generando una ER de bajo impacto.

\section{Discusión y conclusiones}

Muchos de los problemas identificados en el sistema de ER del Maga en los departamentos de Chiquimula y Zacapa también han sido observados en otros países. En este sentido, Landini (2012a) también señaló el rol estructurante que tiene la dinámica político-institucional y, en particular, la politización de la Dirección de Extensión Agraria (DEAg) en las prácticas de ER de Paraguay, mientras que Rivera (2002) mencionó la existencia de una cultura política en Costa Rica donde los nombramientos de extensionistas estaban condicionados por intereses políticos. Ahora bien, teniendo en cuenta la amplitud de la corrupción política y el clientelismo en América Latina, llama la atención que no sea una problemática que se mencione con más frecuencia en los estudios especializados, situación que podría estar evidenciando cierta dificultad para comprender su potencial incidencia en los servicios de ER públicos de diferentes países, o incluso el temor de hablar o visibilizar la problemática por parte de los propios extensionistas. Como señala Landini (2012b, p. 219), no es infrecuente que sujetos enmarcados en contextos y prácticas clientelares recurran a la autocensura: "quienes saben que pueden ser objeto de una amenaza o aun de que ésta pueda hacerse efectiva, procuran guardar silencio y mantener un perfil bajo".

En cuanto a la debilidad del Maga, Sepulcri \& Paula (2008) también han hecho referencia a la fragilidad institucional de otra institución pública latinoamericana, la Empresa Paranaense de Asistencia Técnica y Extensión Rural del Estado de Paraná, Brasil. En la misma línea, otros autores han hecho referencia a factores que contribuyen a la debilidad de instituciones de ER similares a los observados en el MAGA. Así, tanto Matthew \& Olatunji (2016) en Nigeria, como Landini (2012a) en Paraguay, han llamado la atención sobre los problemas generados por la existencia de lineamientos institucionales de ER inestables y cambiantes. A la vez, Ardila (2010) también ha señalado que en América Latina las condiciones laborales de los extensionistas no suelen ser buenas, observándose en muchos casos la contratación por proyectos o por períodos limitados, situación que no permite la consolidación de un equipo estable y tiende a la pérdida de conocimiento por la alta rotación de personal (Brito et al., 2012). En la misma línea, al igual que se observó en el caso estudiado, en la literatura académica sobre ER también se menciona frecuentemente que la formación de los extensionistas tiende a ser en general limitada, tanto a nivel de formación básica como en lo referido a la formación en servicio (Aguirre, 2012; Rodríguez et al., 2016; Landini et al., 2017).

Finalmente, en cuanto a los productores, diversos autores han señalado la existencia de dificultades para lograr la consolidación de grupos o de entidades de productores en el marco del trabajo de ER (Boas \& Goldey, 2005; Landini, 2016b), así como la existencia de actitudes pasivas, asistencialistas o poco participativas por parte de los beneficiarios, muchas veces poco comprometidos con los objetivos de largo plazo que les proponen los extensionistas (Boas \& Goldey, 2005; Landini, 2010; Rivas et al., 2010). 
Luego del análisis descriptivo de los diferentes problemas identificados y su comparación con otros estudios y países, resulta de interés plantear dos reflexiones que atraviesan transversalmente este estudio. La primera hace referencia a la tensión entre la determinación de las estructuras y la agencia de los sujetos (Long, 2001) o, en este caso, el grado en que las estructuras institucionales y políticas del Maga condicionan las prácticas de extensión y el margen de maniobra que tienen los extensionistas y otros actores, tanto para transformar estas estructuras como para generar impactos transformadores en los territorios. En este sentido, conviene señalar, en línea con Lapalma (2001), que los actores siempre tienen tanto un ámbito en el que pueden incidir a partir de sus alianzas y sus recursos de poder disponibles, como un contexto que excede a su capacidad de acción e influencia. En términos del estudio realizado, esto implica que los actores (en este caso extensionistas, funcionarios, niveles decisorios institucionales y organizaciones interesadas en fortalecer el impacto de la extensión rural) siempre tienen un margen de influencia que corresponde a su nivel de acción, por lo que no existen razones objetivas para caer en la actitud desesperanzada de que nada puede hacerse. No obstante, también se reconoce la importancia de una perspectiva realista que reconozca que los cambios posibles dependen del nivel en que se encuentren los diferentes actores y de las alianzas que estos puedan establecer, por lo que habrá que discernir en cada situación concreta y para cada actor particular en qué será posible incidir.

En paralelo, el estudio también mostró que diferentes procesos de orden político o institucional, como ser el elevado grado de politización del contexto guatemalteco o la debilidad institucional del Maga, tienden a generar impactos a nivel psicosocial o actitudinal, como la pérdida de compromiso de los extensionistas o la adopción de una actitudes asistencialistas o cortoplacistas por parte de los productores (Landini et al., 2014). En este sentido, es importante notar que este trabajo plantea que se trata de efectos emergentes de los marcos institucionales y no problemas de carácter primario. Es decir, se argumenta que, en buena medida, la pérdida de compromiso de los extensionistas y las actitudes asistencialistas de los productores no pueden interpretarse como hechos primarios, independientes de su contexto, sino que deben pensarse, precisamente, como su consecuencia. Claro está, esto no quita que luego, una vez instalados dichos impactos en los sujetos, se generen procesos de retroalimentación, en los cuales actitudes de falta de compromiso (en los extensionistas) o de carácter asistencialista (en los productores), retroalimenten las condiciones que los produjeron, como cuando los extensionistas dejan de buscar fortalecer su propia institución y se adaptan a la lógica política de selección de personal, o cuando los productores reclaman beneficios de corto plazo en el vínculo con los extensionistas más que apoyo de largo plazo. En este marco, se destaca la importancia de reconocer el carácter sistémico de los problemas y no entenderlos de manera independiente, en particular aquellos que hablan sobre las actitudes de los propios sujetos.

Respecto de las contribuciones específicas de este trabajo, en primer lugar, se destaca el ofrecer una mayor comprensión de los problemas enfrentados por el sistema de ER del Maga en el oriente guatemalteco, lo que facilitará la tarea de generar acciones tendientes a fortalecer la institución y mejorar sus impactos. A la vez, indirectamente, estos resultados también brindan inspiración e ideas para reflexionar sobre las problemáticas de otras instituciones de ER latinoamericanas, en tanto muchos de los problemas identificados, como se ha evidenciado, se repiten en otros contextos. Ahora bien, en contraste con lo que se observa en la mayor parte de los estudios sobre problemas de la ER en América Latina, los cuales suelen abordan dificultades puntuales o específicas, se destaca que el presente trabajo aporta una visión integrada y sistémica, lo que permite una comprensión más profunda de la integralidad del entramado problemático a abordar, visibilizándose con mayor facilitad las relaciones causales existentes entre los diferentes problemas.

A la vez, a partir de adoptar esta mirada sistémica, el presente estudio también permite analizar la importancia relativa de los problemas identificados. En este sentido, se destaca la incidencia de la politización y la corrupción política como componente estructurante de la problemática observada, algo que podría presumirse que tiene relevancia en otros territorios y países, pero que no suele ser mencionado en la literatura académica. En segundo lugar, y esta vez sí en paralelo con lo que suelen señalar los especialistas, se observa la gran incidencia que tiene la debilidad de la institución que lleva adelante acciones de ER (en este caso el Maga) 
tanto a nivel de los extensionistas (escasa formación, limitado compromiso, cultura de la simulación) como en la obtención de escasos impactos a nivel de los productores.

Por último, a diferencia de los trabajos que abordan los sistemas de extensión a partir de su estructura formal y de los procedimientos establecidos en sus políticas (véase por ejemplo Aguirre, 2012), el presente artículo muestra el interés de estudiar cómo funcionan realmente estos sistemas, en lugar de asumir que las prácticas se identifican con lo que indica la documentación institucional. De hecho, en este trabajo se hizo evidente la gran diferencia existente entre lo que se supone que debería hacerse y lo que realmente se hace, lo que invita a priorizar estudios de campo en contraste con lo que son trabajos que sólo focalizan en el análisis de políticas.

Por su parte, ante el entramado problemático identificado pueden plantearse dos recomendaciones centrales. Por un lado, asumiendo que es limitada la influencia para transformar la 'cultura política' del contexto, parece recomendable llevar a cabo acciones que permitan fortalecer el sistema de ER del Maga, fundamentalmente a partir de generar sistemas de contratación por períodos razonables que utilicen procedimientos objetivos, e implementar procesos de seguimiento y evaluación que contribuyan tanto a tomar decisiones sobre los recursos humanos como a avanzar hacia una mejora institucional continua. A la vez, en cuanto a la falta de insumos y recursos operativos, si no están disponibles en la propia institución, se debe facilitar la articulación con otras instituciones que sí dispongan de estos recursos pero tengan escasez de personal técnico. Por último, también resulta recomendable diseñar estrategias de formación de extensionistas, que incluyan desde capacitaciones en servicio (aprovechando los recursos internos y externos disponibles), hasta la institucionalización de metodologías para formar extensionistas apoyadas en el intercambio entre pares y en el acompañamiento de nuevos extensionistas por parte de los más experimentados.

En cuanto a las limitaciones de este estudio, es importante señalar que se trata de un trabajo cualitativo centrado en una muestra de extensionistas que trabajan en Chiquimula y Zapaca, dos departamentos localizados al oriente del país, por lo que no puede considerarse que estos resultados describan necesariamente lo que sucede a nivel país. En esta línea, se hace evidente el valor complementar y ampliar esta investigación con estudios futuros. En particular, se destaca el interés de realizar trabajos cuyas muestras retomen la amplitud y diversidad territorial del país, e incorporen como entrevistados a agricultores beneficiados por el trabajo de extensión del Maga. Por su parte, también se reconoce la importancia de complementar los resultados cualitativos obtenidos con estudios de tipo cuantitativo, por ejemplo, para cuantificar la importancia relativa de los diferentes problemas y precisar sus relaciones causales. Por último, también se recomienda la realización de investigaciones que aborden el impacto del trabajo de extensión (Christoplos et al., 2012), relacionándolo con los problemas de nivel institucional y de implementación analizados en este trabajo.

\section{Agradecimientos}

Los autores agradecen tanto a Global Affairs Canada como al Consejo Nacional de Investigaciones Científicas y Técnicas de Argentina por la financiación de este estudio, el primero en el marco del "Programa de acceso equitativo a los servicios de salud y la seguridad nutricional" (SETH) liderado por la Fundación Acción Contra el Hambre, y el segundo en el contexto del Proyecto de Investigación Plurianual 112-201501-00108-CO.

\section{Referencias}

Aguirre, F. (2012). El nuevo impulso de la extensión rural en América Latina. Situación actual y perspectivas. Santiago de Chile: RELASER.

Anderson, J., \& Feder, G. (2004). Agricultural extension: Good intentions and hard realities. The World Bank Research Observer, (19), 41-60.

Ardila, J. (2010). Extensión rural para el desarrollo de la agricultura y la seguridad alimentaria: Aspectos conceptuales, situación y una visión de futuro. San José: IICA. 
Baiardi, A., \& Alencar, C. (2014). Agricultura familiar, seu interesse acadêmico, sua lógica constitutiva e sua resiliência no Brasil. Revista de Economia e Sociologia Rural, 52(Supl. 1), 45-62.

Belik, W. A. (2015). A Heterogeneidade e suas implicações para as políticas públicas no rural brasileiro. Revista de Economia e Sociologia Rural, 53(1), 9-30.

Boas, A., \& Goldey, P. (2005). A comparison on farmers' participation in farmers' organizations and implications for rural extension in Minas Gerais. Organizações Rurais y Agroindustriais, 7(3), 259-270.

Brito, L., Souza, P., \& Cartaxo, A. (2012). Gestão do conhecimento numa instituição pública de assistência técnica e extensão rural do Nordeste do Brasil. Revista de Administração Pública, 46(5), 1341-1366.

Cárdenas Hernández, J. (2014). Caracterización del Sistema Nacional de Extensión Rural en Guatemala, departamentos de Chimaltenango, Baja Verapaz y Alta Verapaz (Tesis de máster). Centro Agronómico Tropical de Investigación y Enseñanza, Cartago, Costa Rica.

Christoplos, I., Sandison, P., \& Chipeta, S. (2012). Guide to evaluating rural extension. Lindau, Suiza: GFRAS.

Food and Agriculture Organization - FAO, \& Pan American Health Organization - OPS. (2017). 2017 panorama de la seguridad alimentaria y nutricional en América Latina y el Caribe. Recuperado el 6 de marzo de 2018, de http://www.fao.org/3/a-i7914s.pdf

Food and Agriculture Organization - FAO. (2010). Corporate strategy on capacity development. Recuperado el 6 de marzo de 2018, de www.fao.org/fileadmin/user_upload/newsroom/docs/Summary_Strategy_PR_E.pdf

Forrest, J. (2017). Rural development and food security in the 21st Century: A review and proposal. Journal of Developing Societies, 33(4), 448-468.

Guatemala. Ministerio de Agricultura, Ganadería y Alimentación de Guatemala - MAGA. (2018). Recuperado el 6 de marzo de 2018, de www.maga.gob.gt

Holt Giménez, E. (2008). Campesino a campesino. Voces de Latinoamérica - Movimiento Campesino para la Agricultura Sustentable. Managua: SIMAS.

Klerkx, L., De Grip, K., \& Leeuwis, C. (2006). Hands off but strings attached: the contradictions of policyinduced demand-driven agricultural extension. Agriculture and Human Values, 23(2), 189-204.

Knierim, A., Labarthe, P., Laurent, C., Prager, K., Kania, J., Madureira, L., \& Ndah, T. (2017). Pluralism of agricultural advisory service providers - Facts and insights from Europe. Journal of Rural Studies, 55, 45-58.

Landini, F. (2010). Ingenieros extensionistas formoseños desde la mirada de los pequeños productores. Representaciones, expectativas y realidades. Mundo Agrario, 10(20), 1-23. Recuperado el 15 de marzo de 2018, de www.mundoagrario.unlp.edu.ar/article/view/v10n20a06/451

Landini, F. (2012a). Problemas en la extensión rural paraguaya: Modelos de extensión en la encrucijada. Cuadernos de Desarrollo Rural, 9(69), 127-149.

Landini, F. (2012b). Prácticas clientelares y control político en la experiencia campesina de Argentina. Perfiles Latinoamericanos, 20(40), 205-226.

Landini, F. (2016a). How to be a good rural extensionist. Reflections and contributions of Argentine practitioners. Journal of Rural Studies, 43, 193-202.

Landini, F. (2016b). Problemas de la extensión rural en América Latina. Perfiles Latinoamericanos, 24(47), 47-68.

Landini, F., Brites, W., \& Mathot, M. (2017). Towards a new paradigm for rural extensionists' in-service training. Journal of Rural Studies, 51, 158-167.

Landini, F., Long, N., Leeuwis, C., \& Murtagh, S. (2014). Theoretical guidelines for a psychology of rural development. Cuadernos de Desarrollo Rural, 11(74), 125-147.

Lapalma, A. (2001). El escenario de la intervención comunitaria. Revista de Psicología de la Universidad de Chile, 10(2), 61-70.

Leeuwis, C. (2004). Communication for rural innovation. Rethinking agricultural extension. Oxford: Blackwell Science.

Long, N. (2001). Development sociology. Actor perspectives. Londres: Routledge.

Matthew, A., \& Olatunji, O. (2016). Policy issues for improving monitoring and evaluation of agricultural extension programmes in Nigeria. African Evaluation Journal, 4(1), 1-5. 
Ortiz, R., Rivera, O., Cifuentes, I., \& Morrás, E. (2011). Estudio de sistematización de buenas prácticas de extensión en Guatemala. Guatemala: FAO.

oTranscribe. (2018). Recuperado el 6 de marzo de 2018, de www.otranscribe.com

Rivas, A., Avendaño, P., \& Quintero, H. (2010). Updating peasant competencies to mitigate poverty in the Chorti community, Copan (Honduras). Agronomia Colombiana, 28(3), 567-575.

Rivera, F. (2002). Una aproximación al desarrollo rural de Costa Rica. La extensión agrícola: breve síntesis de su evolución y tendencias. Educare (San José), (3), 29-43.

Robirosa, M., Cardarelli, G., \& Lapalma, A. (1990). Turbulencia y planificación social. Buenos Aires: UNICEF-Siglo XXI

Rodríguez, H., Ramírez, C., \& Restrepo, L. (2016). Nuevas tendencias de la extensión rural para el desarrollo de capacidades de autogestión. Corpoica, 17(1), 31-42.

Sæther, B. (2010). Agricultural extension services and rural innovation in inner Scandinavia. Norsk Geografisk Tidsskrift, 64(1), 1-8.

Sepulcri, O., \& Paula, N. (2008). O Estado e seus impactos na Emater-Pr. Revista Paranaense de Desenvolvimento, (114), 87-110.

Sulaiman, R., \& Davis, K. (2012). The "new extensionist": roles, strategies, and capacities to strengthen extension and advisory services. Lindau, Suiza: GFRAS.

Taylor, S., \& Bodgan, R. (1987). Introducción a los métodos cualitativos de investigación. Barcelona: Paidós.

Zwane, E. (2012). Does extension have a role to play in rural development? South African Journal of Agricultural Extension, 40(1), 16-24.

Data de submissão: 6 de março de 2018.

Data de aceite: 22 de dezembro de 2018

Clasificación JEL: Q16. 